\title{
, \\ Accuracy and Precision in Electronic Structure Computation: Wien2k and FPLO
}

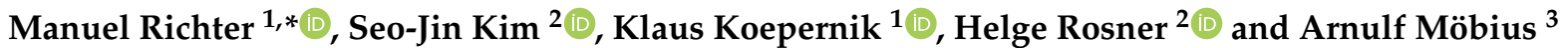 \\ 1 Institute for Theoretical Solid State Physics, Leibniz IFW Dresden, Helmholtzstr. 20, \\ D-01069 Dresden, Germany; k.koepernik@ifw-dresden.de \\ 2 MPI-CPfS Dresden, Nöthnitzer Str. 40, D-01187 Dresden, Germany; seojin.kim@cpfs.mpg.de (S.-J.K.); \\ rosner@cpfs.mpg.de (H.R.) \\ 3 Institut für Physik, Technische Universität Chemnitz, D-09107 Chemnitz, Germany; \\ arnulf.moebius@t-online.de \\ * Correspondence: m.richter@ifw-dresden.de
}

check for updates

Citation: Richter, M.; Kim, S.-J.; Koepernik, K.; Rosner, H.; Möbius, A. Accuracy and Precision in Electronic Structure Computation: Wien2k and FPLO. Computation 2022, 10, 28 https://doi.org/10.3390/ computation 10020028

Academic Editor: Henry Chermette

Received: 6 January 2022

Accepted: 7 February 2022

Published: 11 February 2022

Publisher's Note: MDPI stays neutral with regard to jurisdictional claims in published maps and institutional affiliations.

Copyright: (C) 2022 by the authors. Licensee MDPI, Basel, Switzerland. This article is an open access article distributed under the terms and conditions of the Creative Commons Attribution (CC BY) license (https:// creativecommons.org/licenses/by/ $4.0 /)$.

\begin{abstract}
Electronic structure calculations in the framework of density functional theory are based on complex numerical codes which are used in a multitude of applications. Frequently, existing experimental information is used as a gauge for the reliability of such codes. However, their results depend both on the chosen exchange-correlation energy functional and on the specific numerical implementation of the Kohn-Sham equations. The only way to disentangle these two items is a direct comparison of two or more electronic structure codes. Here, we address the achievable numerical accuracy and numerical precision in the total energy computation of the two all-electron density-functional codes Wien2k and FPLO. Both codes are based on almost independent numerical implementations and largely differ in the representation of the Bloch wave function. Thus, it is a highly encouraging result that the total energy data obtained with both codes agree within less than $10^{-6}$. We here relate the term numerical accuracy to the value of the total energy $E$, while the term numerical precision is related to the numerical noise of $E$ as observed in total energy derivatives. We find that Wien $2 \mathrm{k}$ achieves a slightly higher accuracy than FPLO at the price of a larger numerical effort. Further, we demonstrate that the FPLO code shows somewhat higher precision, i.e., less numerical noise in $E$ than Wien2k, which is useful for the evaluation of physical properties based on derivatives of $E$.
\end{abstract}

Keywords: density functional theory; DFT codes; electronic structure calculation; numerical accuracy and precision

\section{Introduction}

Computations of electronic structure provide an important input to research in physics, chemistry, and materials science, and density functional theory (DFT) is behind a major part of such computations. While DFT is a formally exact theory, its practical use relies on the implementation of approximative exchange-correlation functionals. A second but not less important ingredient to DFT applications consists in the development and verification of numerical electronic structure codes. Here, we consider the latter subject.

The task to solve the Kohn-Sham equations of DFT is well defined, but its solution is far from being trivial. The mathematical complexity of this system of non-linear integrodifferential equations is mirrored by the complexity of its solvers, numerical codes with typically several $10^{5}$ source lines. Codes of such a size and complexity bear two unavoidable problems: approximations and coding errors. Fortunately, a large number of codes has been developed in past decades by independent teams. These codes partly rely on completely different approximations for, e.g., the representation of the wave function. Thus, many of them can be considered as virtually independent implementations with little risk to suffer from the same source of inaccuracy. Hence, deviations from the numerically exact solution 
can be considered as code-specific and uncorrelated, at least for codes with different wave function representations. As noteworthy exceptions, we mention standard algebra routines that are frequently taken from common software packages and methods for $k$-space integrations. The latter are usually implemented independently in each code but may lead to method-specific convergence behavior with the number of $k$-points.

In 2016, a comparison was published regarding the volume-dependent total energy, $E(V)$, calculated by means of 15 different DFT codes for 71 elements of the periodic table [1]. The goal of that work was to evaluate the achievable precision of Kohn-Sham solvers under well-defined common choices of exchange-correlation functional and treatment of relativistic effects. Thereby, precision was defined as the scatter among computed equationof-state data, in distinction to accuracy which would describe the deviation of the mean DFT result from experiment [1].

In the present work which is again solely focused on the comparison of computations, we will use the terms accuracy and precision in a slightly different sense. Motivated by the idea that the total energy is a variational quantity, lower total energy will be identified with higher numerical accuracy in the common meaning of a smaller systematic error [2]. This choice comes with the known caveat that numerical problems like basis set overcompleteness can reduce the resulting total energy in an unpredictable manner [3] and have to be excluded by appropriate data analysis. Furthermore, our definition only makes sense if all calculations make use of one and the same exchange-correlation approximation. With this condition fulfilled, the numerical accuracy of a modern DFT code is mainly determined by the completeness of the basis set for the representation of the Bloch states.

Regarding precision as a description of random errors [2], we will associate this term with numerical noise along the $E(V)$ curve. This choice is motivated by the following consideration. DFT computations are usually quasi-deterministic. Thus, it is not possible to perform a statistical analysis for a single point on $E(V)$. However, different points on $E(V)$ are known to show quasi-random deviations from averaging approximants to $E(V)$, such as polynomial fits. The default numerical settings of advanced codes are nowadays tight enough to keep these quasi-random contributions to $E(V)$ well below its systematic deviations from a hypothetical, exact solution of the Kohn-Sham problem. In other words, the numerical precision of $E(V)$ is much higher than its numerical accuracy. However, this may not be the case for derivatives of the total energy which are of interest on their own. For the sake of brevity, we will drop the qualifier "numerical" for the considered accuracy and precision in most places below.

Seven of the codes taken into account in the abovementioned comparison are socalled all-electron codes which do not rely on approximative pseudopotentials but include the complete nuclear potential and the electronic core wave functions in the calculation. Considering the scatter of results only among these seven codes used with their numerically best settings, the data produced by Wien2k [4] turned out to be close to the barycenter [1]. This finding confirms the status of Wien2k as the "gold standard" of DFT codes: a welldeserved result of several decades of dedicated work by the developer team of Wien2k, formed and headed by Karlheinz Schwarz.

The predictive computation of physical properties by means of DFT codes has been widely established in past decades. If applied to a large set of materials, such a property screening is faced with resource considerations. Disregarding so-called $O(N)$ methods they are not among the most commonly used DFT codes and are often geared towards certain subsets of compounds like insulators -, the required CPU time and main storage grow with the third and second power, respectively, of the Hamilton matrix rank. Hence, a code with a small matrix, i.e., a small basis set, is usually superior to a code with a large basis set in terms of computation time and main storage demand. However, small basis sets frequently limit the achievable accuracy. Thus, it is important to gauge the quality of codes with small basis sets against a confirmed quality standard such as Wien2k both in terms of accuracy and precision. In the current study, we compare Wien $2 \mathrm{k}$ and the full-potential local-orbital (FPLO) code [5] with each other. They are based on completely 
different concepts to represent the Bloch wave function and the FPLO code is equipped with a much smaller basis set than Wien2k. However, both these codes belong to the all-electron category of electronic structure codes. This allows a direct comparison of their total energies.

To establish a procedure for comparing the quality of two codes concerning their predictive power beyond total energies, we advance from the previous equation-of-state comparison [1] to the consideration of total energy derivatives which give access to more detailed thermodynamic information. For example, the first derivative of the total energy with respect to the magnetization equals the external field necessary to stabilize this magnetization, and a zero second derivative indicates a metamagnetic transition. Yet more subtle is the detection of an electronic topological transition (ETT), also called Lifshitz transition, on the sole basis of total-energy data. For hexagonal Osmium, a previous experimental study claimed visibility of an ETT as a kink in the ratio of lattice parameters $c / a$ vs. pressure $p$ [6]. Theoretical equation-of-state data should in principle allow to identify such a peculiarity. In later work, however, the existence of this particular ETT was doubted, see Ref. [7] and references therein.

Here, we demonstrate for a particular case that a conventional ETT can be detected in the third derivative of $E(a)$ as obtained from current DFT codes, $a$ denoting the lattice parameter. The reader may object that ETTs can simply be found by careful inspection of band structure and density of states. This was indeed our starting point in seeking to distinguish the traces of ETTs from numerical noise. We achieved a substantial reduction of the latter by modifying the default numerical settings for both considered codes. This allowed us to obtain even $d^{3} E / d a^{3}$ relations with high resolution. The obtained precision enabled us to perform convincing Birch-Murnaghan fits using the minimum possible number of data points.

Our exercise focuses on the comparatively simple case of face centered cubic (fcc) Aluminium. This metal was already the target of very early Fermi surface investigations by electronic structure theory [8]. Later, Fermi surface changes under different types of pressure were predicted [9] and an ETT was observed by nuclear magnetic resonance experiments on $\mathrm{Al}$ under hydrostatic pressure [10].

Details of the computational methods are provided in the following Section 2. Results and the related discussion are presented in Section 3, and Section 4 summarizes this work.

\section{Methods}

The Wien2k calculations were performed in the version 19.1 of this code [4]. The parameter $R k_{\max }$ was varied in the range from 4 to 12 , and the muffin-tin sphere radius $R_{\mathrm{mt}}$ was chosen as $2.39 \mathrm{Bohr}$ radii for Aluminium. As convergence conditions, $10^{-6}$ for density and $10^{-8}$ Rydberg for the total energy were used in almost all calculations. The only exception was one data point for $a=0.3646 \mathrm{~nm}, R k_{\max }=8$, for which only a density convergence of $10^{-5}$ could be achieved.

FPLO was used in the version FPLO-18.00-52 [11]. Self-consistency of the iterations was considered to be achieved upon stability of both density and total energy. The default values, $10^{-6}$ for density and $10^{-8}$ Hartree for energy, were taken as related thresholds if not indicated otherwise. A specific modification of the numerical mesh for orbital-potential matrix elements is explained and discussed in Section 3.3.

All calculations, performed with either of the codes, were conducted in a scalar relativistic mode according to Koelling and Harmon [12] and using the generalized gradient approximation (GGA) in the parameterization by Perdew, Burke, and Ernzerhof (PBE) [13]. Integrations in reciprocal space were done with a linear tetrahedron method including Blöchl corrections [14]. It should be noted that broadening techniques are less suited for the detection of ETTs.

The $k$-point numbers of the specific calculations are defined in Section 3 using the following notation: A mesh with $n \times n \times n$ intervals in the full Brillouin zone, the irreducible part of which was used in a self-consistent calculation, is denoted as $\operatorname{scK} n$; a mesh with 
$m \times m \times m$ intervals in the full Brillouin zone, the irreducible part of which was used in a single-step calculation with an input density from a calculation $\mathrm{scK} n$, is denoted as $\mathrm{K} m$-scKn .

Calculations were performed for fcc Aluminium (space group 225) with lattice parameters $a$ between $0.36 \mathrm{~nm}$ and $0.41 \mathrm{~nm}$ in steps of $0.0005 \mathrm{~nm}$. In certain cases, the step size was chosen as $0.0001 \mathrm{~nm}$.

In our evaluation of the $E(a)$ data, numerical differentiation plays an important role. This approach, however, requires a trade-off between the conflicting demands for the best possible resolution and the lowest possible noise. Here, we applied two approaches. The first one, called "direct evaluation" henceforth, uses generalizations of the midpoint formula of differentiation. For a given set of data points with equidistant arguments,

$$
d^{2} E /\left.d a^{2}\right|_{a_{i}}=\left(E\left(a_{i+1}\right)-2 E\left(a_{i}\right)+E\left(a_{i-1}\right)\right) /\left(a_{i}-a_{i-1}\right)^{2}
$$

and

$$
d^{3} E /\left.d a^{3}\right|_{\left(a_{i}+a_{i+1}\right) / 2}=\left(E\left(a_{i+2}\right)-3 E\left(a_{i+1}\right)+3 E\left(a_{i}\right)-E\left(a_{i-1}\right)\right) /\left(a_{i}-a_{i-1}\right)^{3}
$$

were evaluated for $\left(a_{i}-a_{i-1}\right)=0.0005 \mathrm{~nm}$. This value is a carefully chosen compromise between a high noise level for small step sizes and a suppression of physical singularities for large step sizes. A considerable further noise reduction can be achieved by starting from third-order polynomial fits within a moving window. This second approach, called "moving window differentiation" below, was carried out on equidistant data sets with $\left(a_{i}-a_{i-1}\right)=0.0001 \mathrm{~nm}$ and 33 data points in each fit.

\section{Results and Discussion}

\subsection{Effect of the $k-M e s h$ on the Density of States}

The density of states (DOS), $g$, is a primary quantity obtained in any electronic structure calculation on extended systems. It has widely been used for the interpretation of numerous experimental results like photon- or electron-spectroscopy data, thermodynamic properties, or electron-nucleus interaction data. Here, we focus on the DOS at the Fermi level $g\left(e_{\mathrm{F}}\right)$. It is related to the low-temperature specific heat and also to the Knight shift. Last but not least, singularities in the dependence of $g\left(e_{\mathrm{F}}\right)$ on the volume (or lattice parameter, or pressure) signal Lifshitz transitions. The aim of this subsection is to sensitize the reader to the importance of choosing a dense $k$-mesh in order to achieve a precise DOS. Henceforth, we use $g\left(e_{\mathrm{F}}\right)$ as abbreviation for $g\left(e_{\mathrm{F}}(a), a\right)$.

Figure 1 shows $g\left(e_{\mathrm{F}}\right)$ of fcc Aluminium vs. lattice parameter $a$ for different $k$-meshes. Consider the upper panel of the figure first. The default $k$-mesh scK12 (red line) provides a smooth behavior of $g\left(e_{\mathrm{F}}\right)$ in the whole range of the lattice parameter. It does, however, not meet the expectation of a root-like dependence for a nearly-free electron situation. For the next denser mesh scK24, a local maximum of $g\left(e_{\mathrm{F}}\right)$ appears close to $a=0.394 \mathrm{~nm}$. This maximum could be related to an ETT, but the yet denser mesh scK50 produces a much more structured curve with several local maxima or kinks. The majority of these singularities can be assigned to numerical minigaps arising from wrong band connections. Such minigaps are more numerous but smaller in the scK100 results, and they are almost invisible in the scK200 curve.

The latter two data sets each exhibit two kinks that indeed originate from ETTs. They are marked by the vertical lines and named ETT1 and ETT2. Furthermore, data obtained with a very dense $k$-mesh K400-scK200 are presented in the lower panel of the figure and in its inset. The related curve is piece-wise quasi-analytic. Here, another but less prominent Lifshitz transition, ETT0, is recognizable close to $a=0.392 \mathrm{~nm}$, see the inset of Figure 1. 


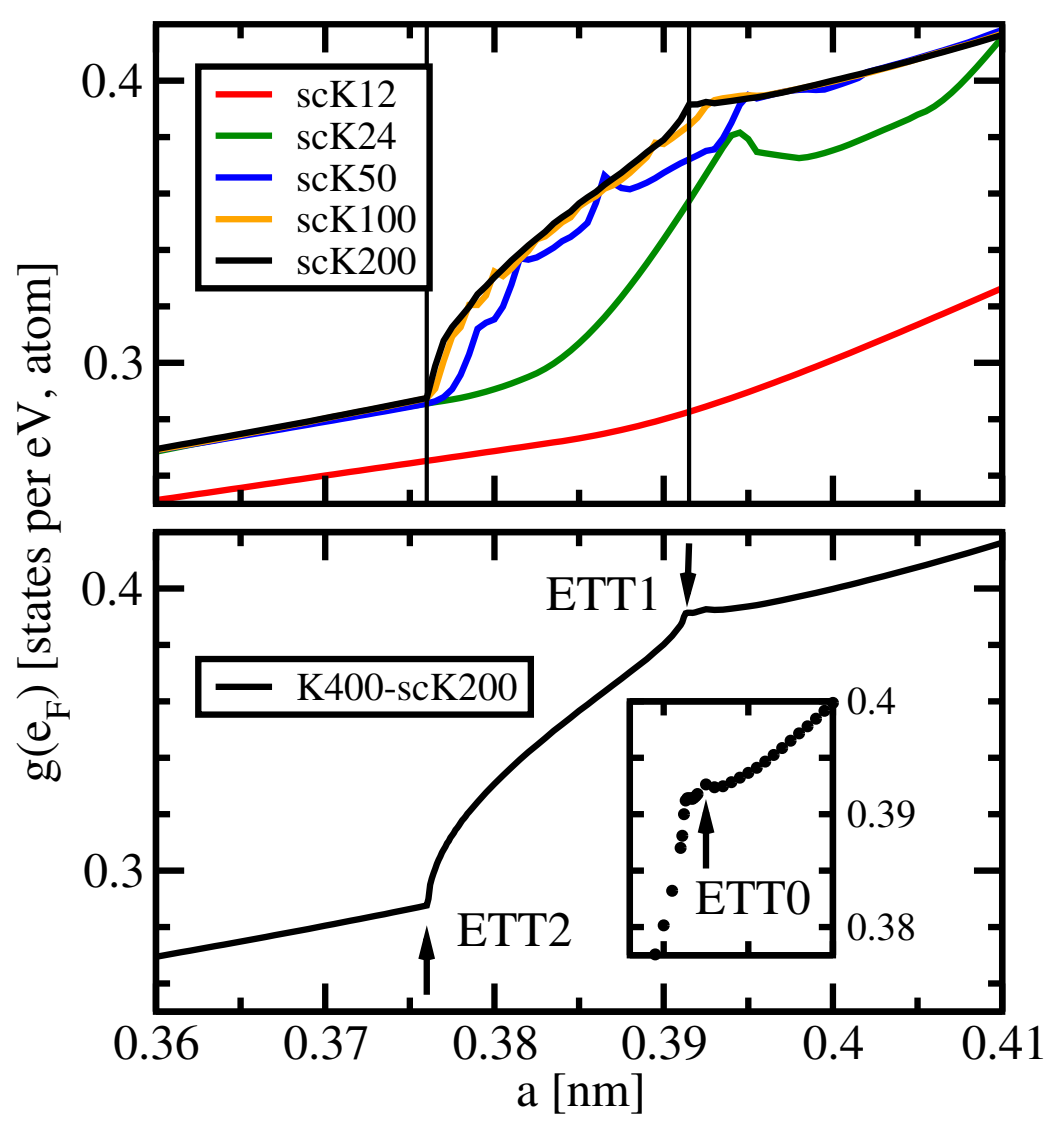

Figure 1. Total density of states at the Fermi level vs. lattice parameter, obtained with the FPLO code using different $k$-meshes as specified in the legends. The positions of two prominent ETTs are indicated by vertical lines in the upper panel and by arrows in the lower panel. Another, less prominent ETT is indicated by an arrow in the inset, which has an $a$-axis identical to that of the main graph. The upper panel shows data points which were obtained at a distance of $0.0005 \mathrm{~nm}$. They are connected by straight lines. The lower panel presents data obtained at the same distance completed by data at a distance of $0.0001 \mathrm{~nm}$ in the vicinity of the ETTs. The data points are connected by lines in the lower main panel and depicted as circles in the inset. The K400-scK200 data were computed with tightened convergence criteria of $10^{-7}$ (density) and $10^{-9}$ Hartree (total energy) in the scK200-step.

All three ETTs detected above can be identified in the schematic Fermi surface plots shown in Figure 2. Therein, the well-known third-zone monster of fcc Aluminium is shown in the left-hand part within the complete Brillouin zone and as the lowest detail in the right-hand part, both for the equilibrium situation.

Consider the right-hand part of Figure 2. Upon reduction of $a$, the $\beta$-orbit merges with its neighbor and the monster become multiply connected (arrow in the second lowest detail). This ETT0 is barely visible in the DOS, compare the inset of Figure 1. A slight further reduction of $a$ disconnects the Fermi surface and lets the $\alpha$-orbits vanish, as shown in the third detail. This transition was named ETT1 above. Finally, the ellipsoid around symmetry point $U$ vanishes in another Lifshitz transition, named ETT2. The latter two transitions are well-resolved in the DOS, provided it is computed with appropriate precision, see Figure 1.

To avoid the occurrence of the abovementioned artificial singularities of the DOS, resulting from wrong band connections, broadening techniques are frequently used for $k$-integration and even for the calculation of the DOS. Such approaches, however, do not allow for the identification of van Hove singularities as indicators of ETTs. Hence, the use of a linear tetrahedron method with a very dense $k$-mesh is the only safe way to identify an ETT in the DOS. An alternative way is to calculate Fermi surfaces using an equally dense mesh. We stress that a band structure at symmetry lines is usually not sufficient for this 
aim since changes of the Fermi surface topology frequently happen at general $k$-points, compare Figure 2.
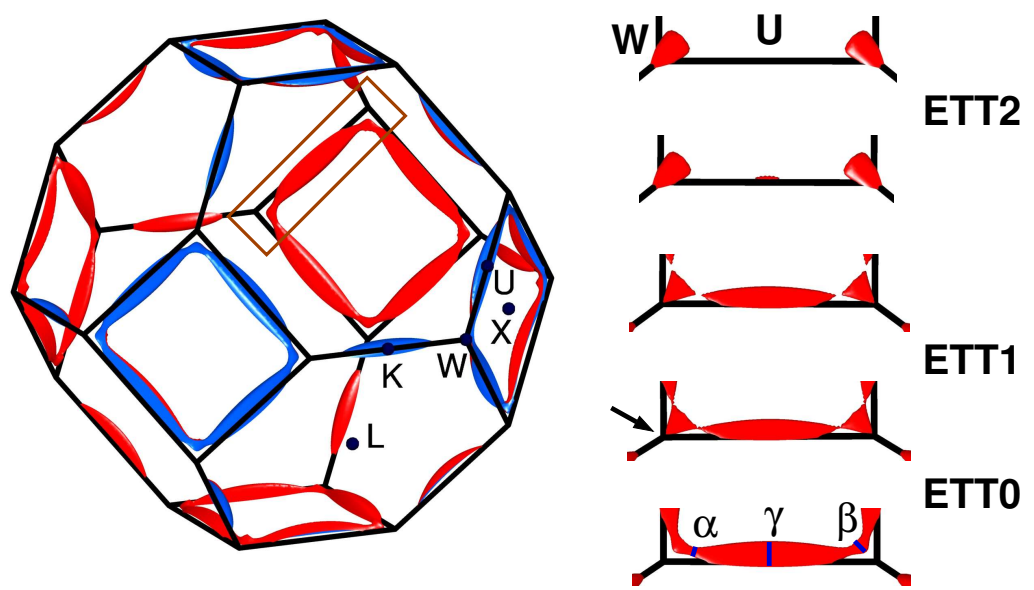

Figure 2. Schematic Fermi surface of Aluminium as obtained with the FPLO code. Left part: Thirdzone equilibrium-volume Fermi surface in the fcc Brillouin zone with symmetry points. Blue (red) color indicates the occupied (unoccupied) side of the Fermi surface. Right part: variation of a detail of the Fermi surface close to one of the square edges as indicated by a brown rectangle in the left part. The lattice parameter shrinks from bottom to top. The lowest detail shows the equilibriumvolume situation with three extremal orbits $(\alpha, \beta, \gamma)$ indicated in the common notation [9] . Our denominations ETT0, ETT1, and ETT2 refer to the Lifshitz transitions detected in Figure 1.

The limited precision of DOS data evaluated by using a coarse $k$-mesh also results in large errors in the predicted values of Sommerfeld parameters, Pauli susceptibilities, and Knight shifts. To illustrate this remark, Table 1 compiles the relative differences between the well-converged value of $g\left(e_{\mathrm{F}}\right)$ obtained from K400-scK200 and $g\left(e_{\mathrm{F}}\right)$ data obtained from less dense meshes for three specific lattice parameters. It shows that the default mesh, which is in many cases sufficient for fairly accurate self-consistent calculations, provides an error of up to $30 \%$ in $g\left(e_{\mathrm{F}}\right)$ at the considered lattice parameters. Even the rather dense mesh scK100 yields an error of up to $2 \%$.

Table 1. Relative differences $1-g\left(e_{\mathrm{F}}\right)_{\mathrm{other}} / g\left(e_{\mathrm{F}}\right)_{400}$ between $g\left(e_{\mathrm{F}}\right)_{400}$, computed from K400-scK200, and $g\left(e_{\mathrm{F}}\right)_{\text {other }}$, computed from other $k$-meshes, for lattice parameters close to the equilibrium structure and close to ETT1 and ETT2. All data were obtained with the FPLO code.

\begin{tabular}{llccccc}
\hline Mesh & scK12 & scK24 & scK50 & scK100 & scK200 \\
\hline $1-g\left(e_{\mathrm{F}}\right)_{\text {other }} / g\left(e_{\mathrm{F}}\right)_{400}$ at $a=0.4040 \mathrm{~nm}$ & 0.234 & 0.051 & -0.0008 & 0.0002 & -0.0002 \\
$1-g\left(e_{\mathrm{F}}\right)_{\text {other }} / g\left(e_{\mathrm{F}}\right)_{400}$ at $a=0.3915 \mathrm{~nm}$ & 0.278 & 0.087 & 0.049 & 0.019 & -0.0004 \\
$1-g\left(e_{\mathrm{F}}\right)_{\text {other }} / g\left(e_{\mathrm{F}}\right)_{400}$ at $a=0.3760 \mathrm{~nm}$ & 0.078 & 0.007 & 0.007 & 0.0008 & 0.0004 \\
\hline
\end{tabular}

The data presented in Table 2 corroborate the above note that $k$-meshes of usual density are sufficient for the computation of integral properties like the equilibrium lattice parameter $a_{0}$. For each of the considered $k$-meshes, $a_{0}$ was obtained as the minimum of a parabola through the three total energy values computed for $a=0.40415,0.40420,0.40425 \mathrm{~nm}$. These lattice parameter values are close to the GGA equilibrium which we identify with $a_{0, \mathrm{scK} 400}=0.4042144 \mathrm{~nm}$, denoting $a_{0}$ computed with scK400. Already for scK36, the relative error $1-a_{0, \mathrm{scKxx}} / a_{0, \mathrm{scK} 400}$ is below $2 \times 10^{-5}$. Note, this precision is somewhat better than the stability of the Aluminium sample lattice parameter in an experiment without explicit and accurate temperature control. For this situation, temperature stability of $1 \mathrm{~K}$ and thermal expansion of $2.3 \times 10^{-5} \mathrm{~K}^{-1}$ are assumed. After increasing the density of $k$-points by a factor of 8 , that is for scK72, the deviation is already reduced to $2.5 \times 10^{-6}$, far better than the achievable precision of any single $\mathrm{X}$-ray experiment. 
Table 2. Relative differences $1-a_{0, \mathrm{scKxx}} / a_{0, \mathrm{scK} 400}$ between equilibrium lattice parameter $a_{0, \mathrm{scK} 400}$, computed from scK400, and $a_{0, \mathrm{scKxx}}$, computed from other $k$-meshes. All data were obtained with the FPLO code.

\begin{tabular}{llllllll}
\hline Mesh & scK12 & scK24 & scK36 & scK48 & scK72 & scK100 & scK200 \\
\hline $10^{5} \cdot\left(1-a_{0, \text { scKxx }} / a_{0, \text { scK400 }}\right)$ & 2.1 & -8.7 & 1.9 & 0.7 & -0.25 & 0.02 & 0.05 \\
\hline
\end{tabular}

\subsection{Preliminary Considerations-Wien $2 k$}

Before a serious numerical investigation by means of any DFT code for a periodic system can be started, two important decisions regarding the numerical settings have to be taken. The first one, choosing the method and mesh density for $k$-space integration, was discussed in the previous subsection. The second one is the choice of the basis set for the Bloch wave function representation. In the Wien $2 \mathrm{k}$ code, the extend of the basis set is encoded in the parameter $R k_{\max }$, the product of the smallest muffin-tin sphere radius and the largest $k$-vector of the plane wave basis set used in the interstitial space. In addition, the number of local orbitals depends on $R k_{\max }$.

In order to check the impact of $R k_{\max }$ on the accuracy, we performed calculations for a number of integer values around the default value $R k_{\max }=7$, using a dense $k$-mesh K100-scK50. Figure 3 shows the total energy vs. $R k_{\max }$ of fcc Aluminium for $a=0.404 \mathrm{~nm}$, which is close to the GGA equilibrium. The default value of $R k_{\max }$ is indicated by a red circle. We note that the total energy diminishes monotonically with growing $R k_{\max }$ between $4 \leq R k_{\max } \leq 12$. Hence, the accuracy improves with growing $R k_{\max }$ at least till $R k_{\max }=12$. The energy seems to be converged within about $0.1 \mathrm{meV}$ for this largest tested basis set. The default setting $R k_{\max }=7$ provides a total energy about $7 \mathrm{meV}$ above the lowest value.

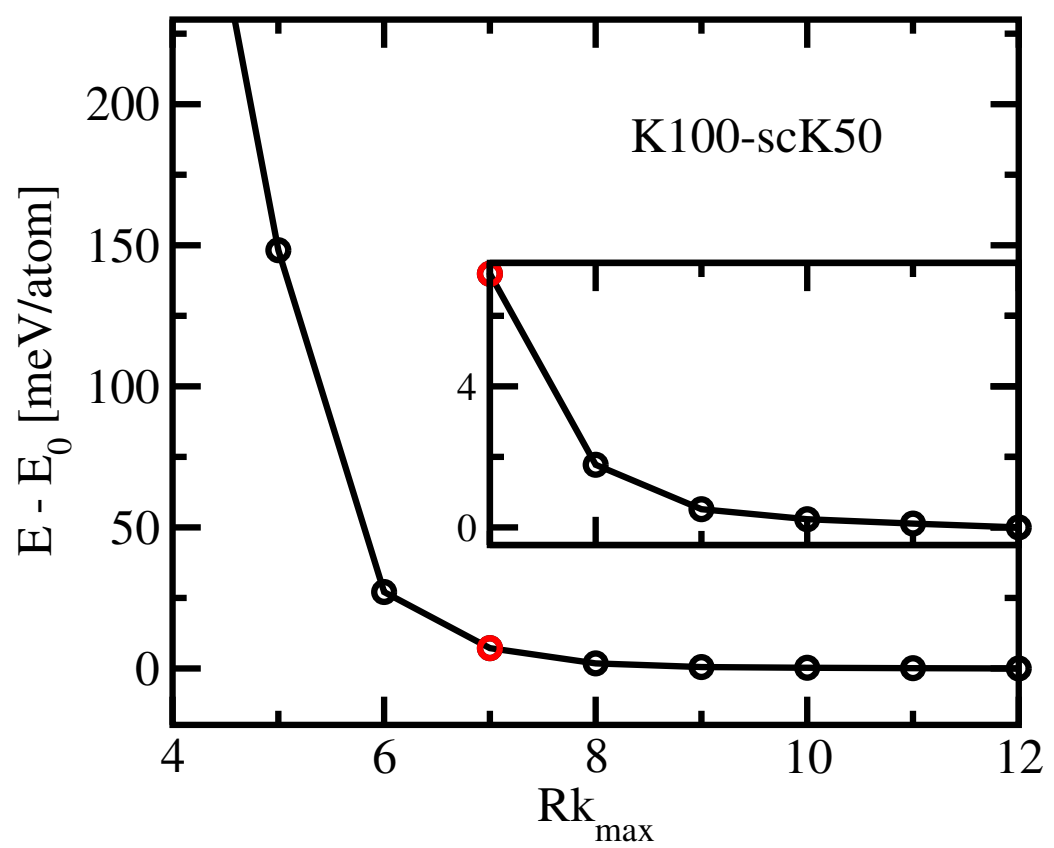

Figure 3. Total energy vs. $R k_{\max }$ of fcc Aluminium for $a=0.404 \mathrm{~nm}$ obtained with the Wien2k code. All data were computed with K100-scK50. The lowest obtained total energy value, $E_{0}=-6,607,524.417 \mathrm{meV} /$ atom, was chosen as reference. The inset shows part of the data using a zoomed energy axis. Its $R k_{\max }$-axis agrees with that of the main plot. Red circles indicate the result for the default value of $R k_{\max }$ and black circles all other calculated points.

Next, we turn to the influence of $R k_{\max }$ on the precision by evaluating total energy derivatives with respect to the lattice parameter. Figure 4 shows $d^{2} E / d a^{2}$ (left panel) and $d^{3} E / d a^{3}$ (right panel) vs. $a$, as obtained using Wien $2 \mathrm{k}$ for a lattice parameter step size of $0.0005 \mathrm{~nm}$. Note that, in order to avoid strong overlap, the individual data sets are 
offset against each other as detailed in the figure caption. For both the second and third derivatives, the numerical noise is smallest for $R k_{\max }=8$ or 9 , slightly above the default value $R k_{\max }=7$. For both $R k_{\max }<8$ and $R k_{\max }>9$, the precision decreases. We stress that, in the case of third derivatives, the noise level is larger than the absolute mean value of the derivative for all values of $R k_{\max }$. This fact will necessitate data smoothing in the search for ETTs, see Section 3.5.

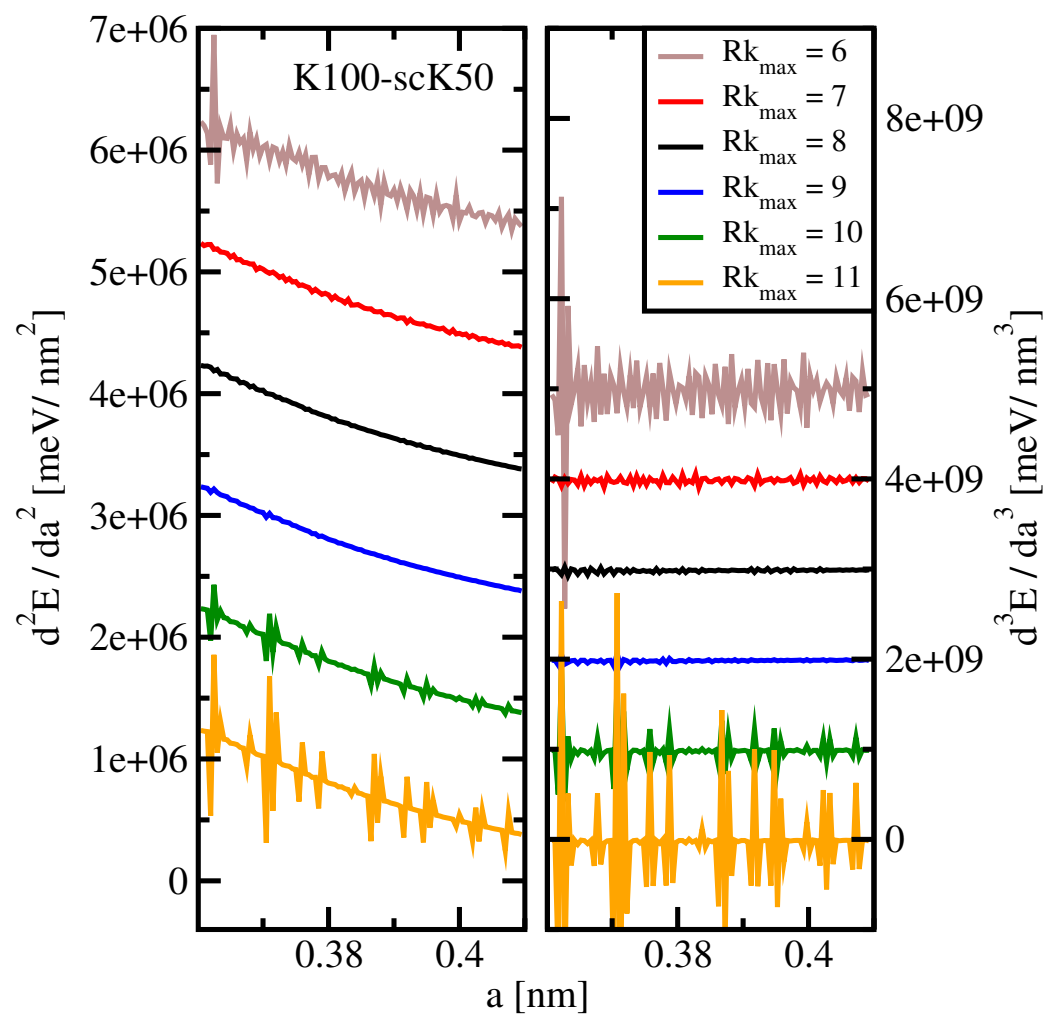

Figure 4. Derivatives of the total energy of fcc Aluminium with respect to the lattice parameter vs. lattice parameter. The total energies were obtained with the Wien $2 \mathrm{k}$ code for different values of $R k_{\max }$ as detailed in the legend. Left panel: second derivative, $d^{2} E / d a^{2}$; the data are offset against the original data by $\left(11-R k_{\max }\right) \cdot 10^{6} \mathrm{meV} / \mathrm{nm}^{2}$. Right panel: third derivative, $d^{3} E / d a^{3}$; the data are offset against the original data by $\left(11-R k_{\max }\right) \cdot 10^{9} \mathrm{meV} / \mathrm{nm}^{3}$. The derivatives are obtained by direct evaluation, see Section 2, for a lattice parameter step size of $0.0005 \mathrm{~nm}$. All data were computed with K100-scK50.

One possible explanation for, above $R k_{\max }=9$, the noise unexpectedly increasing with increasing number of plane waves could be numerical problems arising from near completeness. In such a situation, the newly added basis vectors contain more and more redundant directions in Hilbert space. Shifting (a tiny) wave function weight from one to another of these directions will not alter the charge density in any appreciable manner. However, which directions are finally chosen to contribute to the wave function is increasingly determined by unavoidable numerical noise from various places of the code in a quasi-random fashion, the less important the added directions are. Such noise will reflect in derivatives of the total energy. We have no hypothesis that could explain the observed increase of noise if a smaller basis than the default one is chosen.

\subsection{Preliminary Considerations-FPLO}

The precision of the total energy computation, viz. the noise of $E(V)$, can be evaluated in two ways. In the previous subsection, we used numerical differentiation for this aim. Now, we consider the comparison to a global fit of the $E(V)$ data set: the subtrac- 
tion of a Birch-Murnaghan fit, $E_{\mathrm{BM}-\mathrm{fit}}(V)$, from the original data removes a large and smooth contribution.

Figure 5 compares the results of this procedure when applied to two FPLO data sets obtained with different treatment of orbital-potential matrix elements. In both cases, the rather dense $k$-mesh K200-scK50 and default settings concerning all other parameters were used. The black and red curves present the lattice parameter dependences of the difference $E(a)-E_{\mathrm{BM}-\mathrm{fit}}(a)$ obtained with default and modified integration meshes for the orbital-potential matrix elements, respectively. Along the whole range of considered lattice parameters, these energy differences amount to less than $10^{-7}$ parts of the total energy.

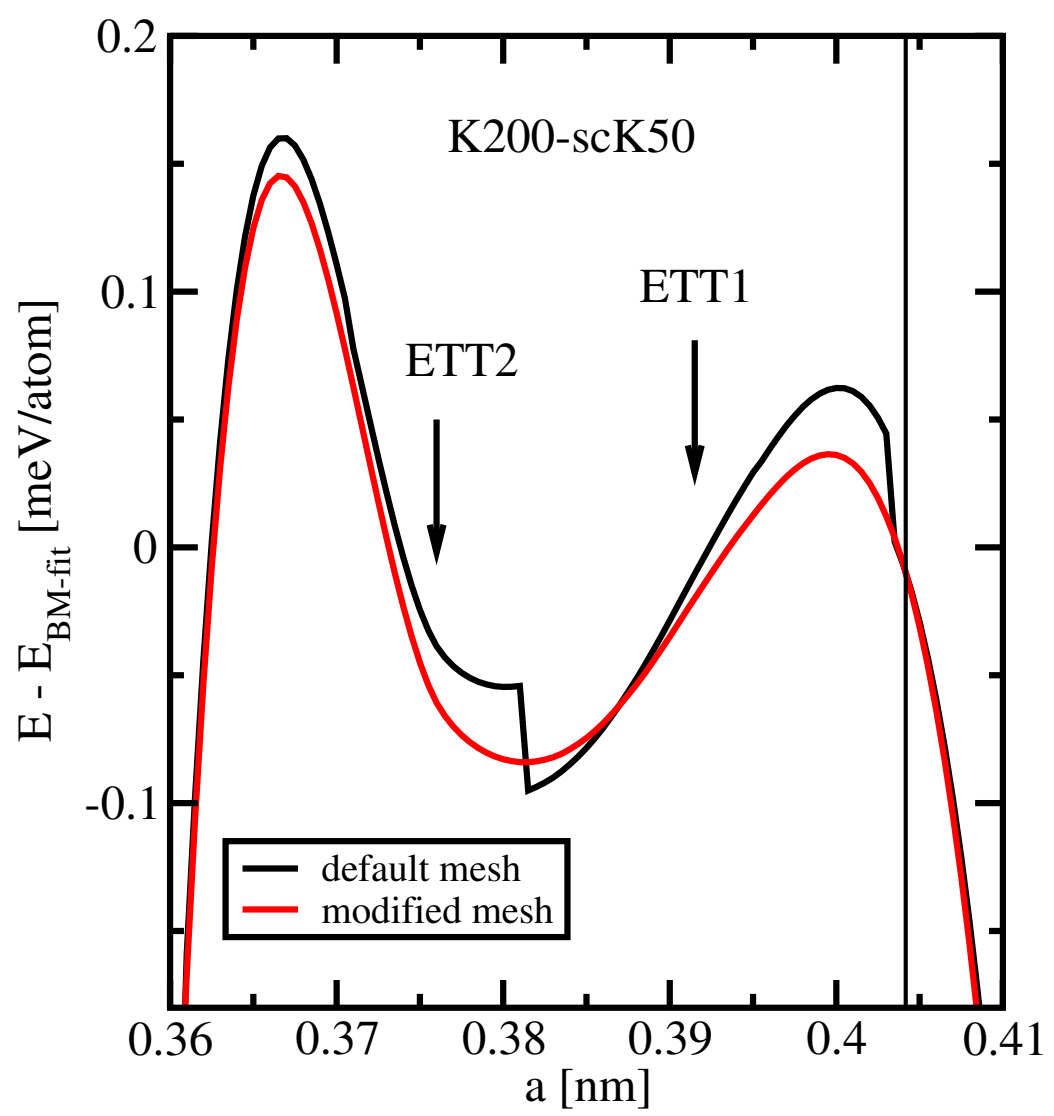

Figure 5. Difference between the total energy calculated with the FPLO code and the total energy obtained with a related third-order Birch-Murnaghan fit vs. lattice parameter. All data were obtained with K200-scK50. The black line shows data obtained with default numerical settings and the red line shows data obtained with a modified mesh for the computation of orbital-potential matrix elements as explained in the text. The arrows indicate the positions of two ETTs, identified in Section 3.1. The vertical line marks the equilibrium lattice parameter derived from the Birch-Murnaghan fit to data obtained with the modified integration mesh.

The general behavior of both $E(a)-E_{\mathrm{BM}-\mathrm{fit}}(a)$ curves is typical of a fourth-order polynomial, as expected for the residual of a third-order fit. However, the black curve exhibits two well-visible discontinuities at $a=0.381 \ldots 0.3815 \mathrm{~nm}$ and $a=0.403 \ldots 0.4035$ $\mathrm{nm}$, as well as another, barely visible one at $a=0.3705 \ldots 0.371 \mathrm{~nm}$. The energy residual $E(a)-E_{\mathrm{BM}-\mathrm{fit}}(a)$ jumps at these lattice parameter values by less than $0.05 \mathrm{meV}$, which is about two orders of magnitude smaller than the energy variations usually encountered. Hence, these discontinuities are not visible in most presentations. They are, moreover, not important in most applications. In the present study, however, they are essential.

A possible idea would be to relate the discontinuities in $E(a)-E_{\mathrm{BM}-\mathrm{fit}}(a)$ to Lifshitz transitions. However, the two ETTs identified in Section 3.1 occur at other lattice parameters, were no singularity in $E(a)-E_{\mathrm{BM}-\mathrm{fit}}(a)$ is visible in Figure 5. The true origin of 
these discontinuities lies in a time-saving approximation in the evaluation of the orbitalpotential matrix elements. This numerical integration is performed on a three-dimensional grid, which combines a radial mesh with angular meshes at each of the radial points. In FPLO, computing time is saved by using angular meshes with lower density for smaller radii. Since, the transition from smaller to larger meshes with varying radius happens at reasonably chosen fixed distances from the nuclei, the number of grid points changes with varying lattice parameter, resulting in the observed discontinuities.

The remedy is to choose the most dense angular mesh at all radii, which increases the CPU time only by about $10 \%$. This modification has been applied to compute the data shown in the red curve of Figure 5. There, no visible deviation from a smooth behavior is observed and the noise level is well below $0.01 \mathrm{meV}$. For this reason, we used the modified mesh for the evaluation of the orbital-potential matrix elements for all calculations presented in this work, except for the default data in Figure 5.

Finally, we remark that such small precision-related effects in the total energy as visualized in Figure 5 can only be detected by considering the difference between total energy and a Birch-Murnaghan or another appropriate polynomial fit. The parameter values of the Birch-Murnaghan fits used here are given in Table 3. They are equivalent to each other, i.e., the precision of these parameters is not reduced by applying the default integration mesh instead of the modified one as long as the fit is performed with a large enough number of data points.

Table 3. Parameter values obtained in the third-order Birch-Murnaghan fits used for Figure 5. $V_{0}$ denotes the equilibrium atomic volume, $B_{0}$ and $B_{0}^{\prime}$ are the bulk modulus and its pressure derivative.

\begin{tabular}{lccc}
\hline Quantity & $\boldsymbol{V}_{\mathbf{0}}\left[\mathrm{nm}^{\mathbf{3}}\right]$ & $\boldsymbol{B}_{\mathbf{0}}[\mathrm{GPa}]$ & $\boldsymbol{B}_{\mathbf{0}}^{\prime}$ \\
\hline Default mesh values & 0.0165056 & 79.05 & 4.29 \\
Modified mesh values & 0.0165050 & 79.06 & 4.29 \\
\hline
\end{tabular}

\subsection{Comparison of Total Energies}

We now compare the accuracies reached by the Wien2k and FPLO codes. This will be done by considering the equation of state for two different basis sets of each code. Figure 6 shows results obtained with default basis sets (Wien2k: $R k_{\max }=7$; FPLO: local states $2 s, 2 p, 3 s, 3 p, 3 d, 4 s, 4 p$ ) and with basis sets for enhanced accuracy (Wien2k: $R k_{\max }=9$; FPLO: local states $2 s, 2 p, 3 s, 3 p, 3 d, 4 s, 4 p, 4 d, 4 f, 5 s, 5 p)$. For the Wien2k code, the enhanced basis set was defined according to the precision considerations in Section 3.2; for the FPLO code, the enhanced basis set as defined in the appendix to Ref. [1] was used. We remark that the $R k_{\max }$ settings for Wien $2 \mathrm{k}$ in the mentioned reference were 6.5 for default and 10.0 for enhanced accuracy calculations [1].

We learn from Figure 6 that the characterization of Wien2k as a highly accurate code is once more confirmed. For the default settings, the ground-state energy obtained by FPLO is about $10 \mathrm{meV}$ per atom above the corresponding value obtained by the Wien2k code; considering the enhanced settings, this difference is reduced to about $4 \mathrm{meV}$ per atom. At lattice spacings much smaller than the equilibrium spacings, the order is, however, reversed for the enhanced settings: the FPLO energy is found to be about 4 meV below the Wien $2 \mathrm{k}$ value (see upper inset). A larger value of $R k_{\max }$, which could be chosen at the price of reduced precision, would not change this picture, since it would reduce the total energy by merely $0.5 \mathrm{meV}$ compared with the value for $R k_{\max }=9$. We remark that the different sequences of the total energy values obtained by Wien $2 \mathrm{k}$ and FPLO for equilibrium spacing, on the one hand, and for much smaller values of $a$, on the other hand, are not very surprising: DFT codes are usually optimized to achieve high accuracy close to the equilibrium state. For the case of high pressure, numerical settings may have to be adapted specifically. 


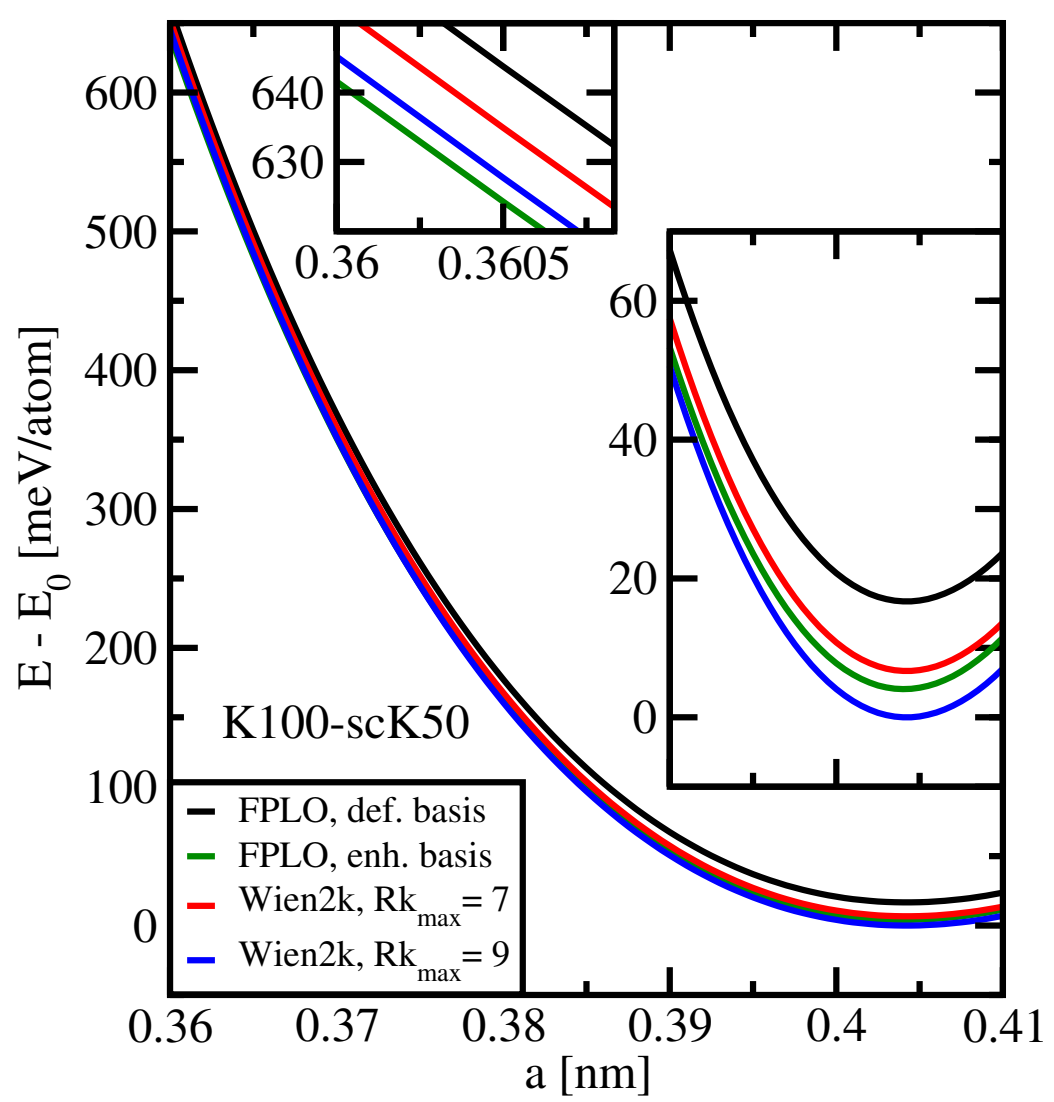

Figure 6. Comparison of total energies of fcc Aluminium vs. lattice parameter calculated with two different codes and two different basis sets for each code as detailed in the legend. All data were obtained with K100-scK50. The lowest total energy, $E_{0}=-6,607,523.903 \mathrm{meV} /$ atom, was chosen as reference. The insets show zoomed regions of the main plot. The $a$-axis of the lower inset agrees with that of the main plot.

Table 4 presents a comparison of the parameter values obtained by means of BirchMurnaghan fits to all four data sets. These fits yield virtually the same results: $a=$ $0.4040 \ldots 0.4042 \mathrm{~nm}$, bulk modulus $B_{0}=79 \mathrm{GPa}$, and its pressure derivative $B_{0}^{\prime}=4.3$.

Table 4. Comparison of third-order Birch-Murnaghan fits applied to the data shown in Figure 6. Rows denoted with $R k_{\max }$ values refer to Wien2k, rows denoted with a basis type refer to FPLO. $V_{0}$ denotes the equilibrium atomic volume, $B_{0}$ and $B_{0}^{\prime}$ are the bulk modulus and its pressure derivative, respectively.

\begin{tabular}{lccc}
\hline Quantity & $\boldsymbol{V}_{\mathbf{0}}\left[\mathbf{n m}^{\mathbf{3}}\right]$ & $\boldsymbol{B}_{\mathbf{0}}$ [GPa] & $\boldsymbol{B}_{\mathbf{0}}^{\prime}$ \\
\hline$R k_{\max }=7$ & 0.0165067 & 79.14 & 4.29 \\
$R k_{\max }=9$ & 0.0165049 & 78.95 & 4.31 \\
\hline default basis & 0.0165050 & 79.06 & 4.29 \\
enhanced basis & 0.0164844 & 78.85 & 4.30 \\
\hline
\end{tabular}

It is very remarkable that two completely independent codes yield total energies with an absolute difference of less than $5 \mathrm{meV}$ and a relative difference of less than $10^{-6}$ along the whole range of lattice parameters. These findings confirms the reliability of both codes because the probability of accidental error compensation is extremely low. Note finally that Aluminium is usually considered to be a nearly-free-electron metal. Thus, it is expected to be best described by plane-wave related methods. The above results prove that, even in this case, a local orbital based code achieves almost the celebrated accuracy of an augmented plane wave code. 
We would like to conclude this evaluation of accuracies with a comparison of the related numerical efforts. One anticipates that a better accuracy requires a larger effort. This expectation is indeed confirmed by the typical computation times presented in Table 5 . We observed that, as a rough estimate, the Wien $2 \mathrm{k}$ calculations consume about one order of magnitude more CPU resources than the FPLO calculations. This difference originates mainly from the following: Wien $2 \mathrm{k}$ uses about twice as many basis functions as contained in the enhanced basis of FPLO.

Table 5. Typical computation times on Intel Xeon CPUs with $2.6 \mathrm{GHz}$ clock frequency to calculate the data shown in Figure 6 . Rows denoted with $R k_{\max }$ values refer to Wien2k, rows denoted with a basis type refer to FPLO. The runtime of each of the cores for one iteration cycle is called $t_{\text {core. }}$ It is given for serial mode ( 1 core) and, in the case of Wien2k, for parallel mode with 16 cores.

\begin{tabular}{lll}
\hline & $\boldsymbol{t}_{\text {core }}[\mathbf{s}] \mathbf{( 1}$ core) & $\boldsymbol{t}_{\text {core }}$ [s] (16 cores) \\
\hline$R k_{\max }=7, \mathrm{scK} 50$ & 21 & 9 \\
$R k_{\max }=7, \mathrm{~K} 100$ & 134 & 20 \\
$R k_{\max }=9, \mathrm{scK} 50$ & 31 & 10 \\
$R k_{\max }=9, \mathrm{~K} 100$ & 190 & 23 \\
default basis, scK50 & 2 & - \\
default basis, K100 & 13 & - \\
enhanced basis, ScK50 & 8 & - \\
enhanced basis, K100 & 48 & - \\
\hline
\end{tabular}

\subsection{Search for ETTs by Means of Total Energy Derivatives}

In Section 3.1, carefully inspecting the lattice parameter dependence of the density of states at the Fermi level, we detected three Lifshitz transitions. Two of them, termed ETT1 and ETT2, are clearly visible in $g\left(e_{\mathrm{F}}\right)$, see Figure 1, provided the $k$-mesh is K100 or better; another one, ETT0, is hardly visible. Close to a three-dimensional ETT related to the bottom of a band at $e_{\mathrm{c}}$, the total energy contains a contribution proportional to $\left(e_{\mathrm{F}}-e_{\mathrm{c}}\right)^{2.5}$ [15]. In other situations, the sign can be changed. Assume linear dependence of $e_{\mathrm{F}}-e_{\mathrm{c}}$ on $a$ close to the transition. Consequently, the ETT should be signaled by a divergence of the third derivative $d^{3} E / d a^{3}$.

We now ask whether the respective optimum total energy precision of Wien2k and FPLO is sufficient for the identification of such divergences. The upper panel of Figure 7 shows $d^{3} E / d a^{3}$ vs. $a$ as obtained by means of both codes with K100-scK50 and subsequent direct numerical differentiation (d). In both cases, the step size of $a$ was set to $0.0005 \mathrm{~nm}$. Despite the use of rather dense $k$-meshes and although the settings of both codes were optimized with respect to low numerical noise, there is no sign of a divergence visible in the upper panel data close to any of the expected critical lattice parameters, which are indicated by vertical lines.

The fluctuations of the Wien2k data (black curve) are considerably stronger than those of the FPLO data (red), which points to a better precision of the latter. We remark that FPLO data generated with enhanced basis set as described in Section 3.4 (not included in Figure 7) exhibit up to four times stronger noise of $d^{3} E / d a^{3}$ than data obtained using the default basis (red curve).

The lower panel of Figure 7 shows data obtained for the setting K200-scK100 using yet denser $k$-meshes than above (blue, Wien2k and orange, FPLO), and for K400-scK200 (green, FPLO). The two former data sets were computed by using a moving window differentiation $(\mathrm{m})$ with 33 equidistant data points (distance $0.0001 \mathrm{~nm}$ ). In this way, a clear singularity is seen at the expected position of ETT2, which is yet more pronounced in the FPLO K400-scK200 data set which was obtained by direct evaluation of the derivative (distance $0.0005 \mathrm{~nm}$ ). On the one hand, the moving window differentiation reduces the numerical noise considerably; on the other hand, it broadens the singularity which is more pronounced in the latter data. Note that the individual data are offset against each other as detailed in the figure caption. 


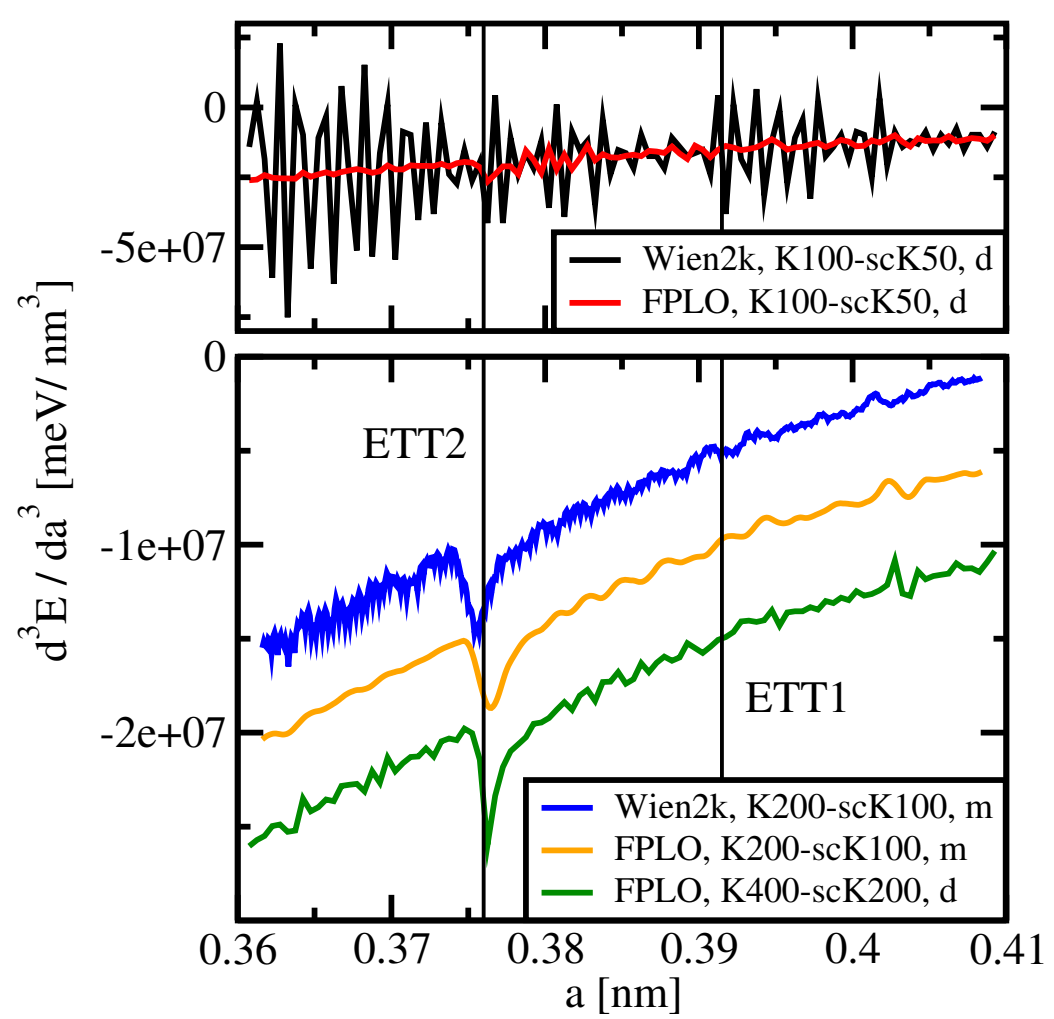

Figure 7. Third derivative of the total energy with respect to lattice parameter $a$ in relation to electronic topological transitions. Upper panel: Comparison of Wien2k data obtained with $R k_{\max }=8$ (same data as shown in the right panel of Figure 4, black curve) with FPLO data (red). Both calculations were performed using the K100-scK50 setting with total energy convergence of $10^{-8}$ Rydberg. The derivatives were obtained by direct evaluation, indicated in the legends by " $\mathrm{d}$ ". Lower panel: Comparison of Wien $2 \mathrm{k}$ data obtained with $R k_{\max }=8$ (blue, with $10^{7} \mathrm{meV} / \mathrm{nm}^{3}$ offset) with FPLO data (orange, with $0.5 \times 10^{7} \mathrm{meV} / \mathrm{nm}^{3}$ offset). Both data sets were obtained using the K200-scK100 setting with total energy convergence of $10^{-8}$ Rydberg. The derivatives were calculated using a moving window with 33 data points, indicated by " $\mathrm{m}$ ", see Section 2. Another FPLO data set, K400-scK200 with total energy convergence of $10^{-9}$ Hartree and direct evaluation of the derivative, is shown in green. The vertical lines indicate the positions of ETT1 and ETT2 as obtained in Section 3.1.

None of the presented data sets shows any peculiarity exceeding numerical fluctuations at the position of ETT1 or of ETT0. We remind that the root-like singularities in $g\left(e_{\mathrm{F}}\right)$ caused by ETT0 and ETT1 are less strong than that one caused by ETT2, see Figure 1. This difference may result from the differing natures of the respective topological changes: ETT0 and ETT1 are the opening and the closing of connections between Fermi surface parts, whereas ETT2 is the vanishing of an isolated part of the Fermi surface, see Figure 2.

To conclude this section, we have shown that an electronic topological transition can in principle be detected in a higher derivative of the total energy. To this end an extreme precision is required, such that the total energy curve appears very smooth as compared to usual code applications. Our calculations have shown that ETT2 is indeed visible as a singularity in $d^{3} E / d a^{3}(a)$, whereas we could not detect the other ETT in this way. The other kinks observed are not related to topological transitions. In consequence, the total energy is not suited to detect such transitions. This implies that similar analyses of experimental data, which have a naturally lower precision than a highly tuned computer code, will be challenging. These strict statements, however, do no longer hold if the ETT is of lower than three-dimensional nature, in which case the transition can become first order and more easily detectable [16]. The differentiation procedures used here are very sensitive and might be helpful both in detecting first or second order phase transitions or in disproving 
their existence, such as in the case of $\beta$-Zirconium. There, contrary to earlier claims, no evidence for isostructural phase transitions was recently found [17].

\subsection{Birch-Murnaghan Fits Using Minimum Number of Data Points}

We have shown in Section 3.1 that the calculation of the equilibrium lattice constant is well converged already for moderately large $k$-meshes. Now, we would like to find out whether it is possible to utilize precise total energy data for evaluating the equilibrium volume, the bulk modulus, and its pressure-derivative with the minimum number of data points needed. For this aim, third-order Birch-Murnaghan fits were performed with only four total energy values obtained using FPLO with K100-scK50 setting. The equidistant fit points are distributed within a fit region of $0.0015 \ldots 0.012 \mathrm{~nm}$ around the equilibrium lattice parameter.

Figure 8 shows results for the three experimentally accessible fit parameters vs. the width of the fit region. For comparison, the results of two Birch-Murnaghan fits to 25 and 13 data points, respectively, are shown as well. As expected from the results presented in Table 2, the equilibrium volume is almost independent of the width of the fit region. Furthermore, the variation of the bulk modulus, $0.1 \mathrm{GPa}$ within the considered range, is negligible. Only the value of $B_{0}^{\prime}$ seems to be unstable if the fit region is chosen smaller than $0.004 \mathrm{~nm}$. This observation is consistent with the fact that the stability of numerical derivatives worsens with their order increasing.

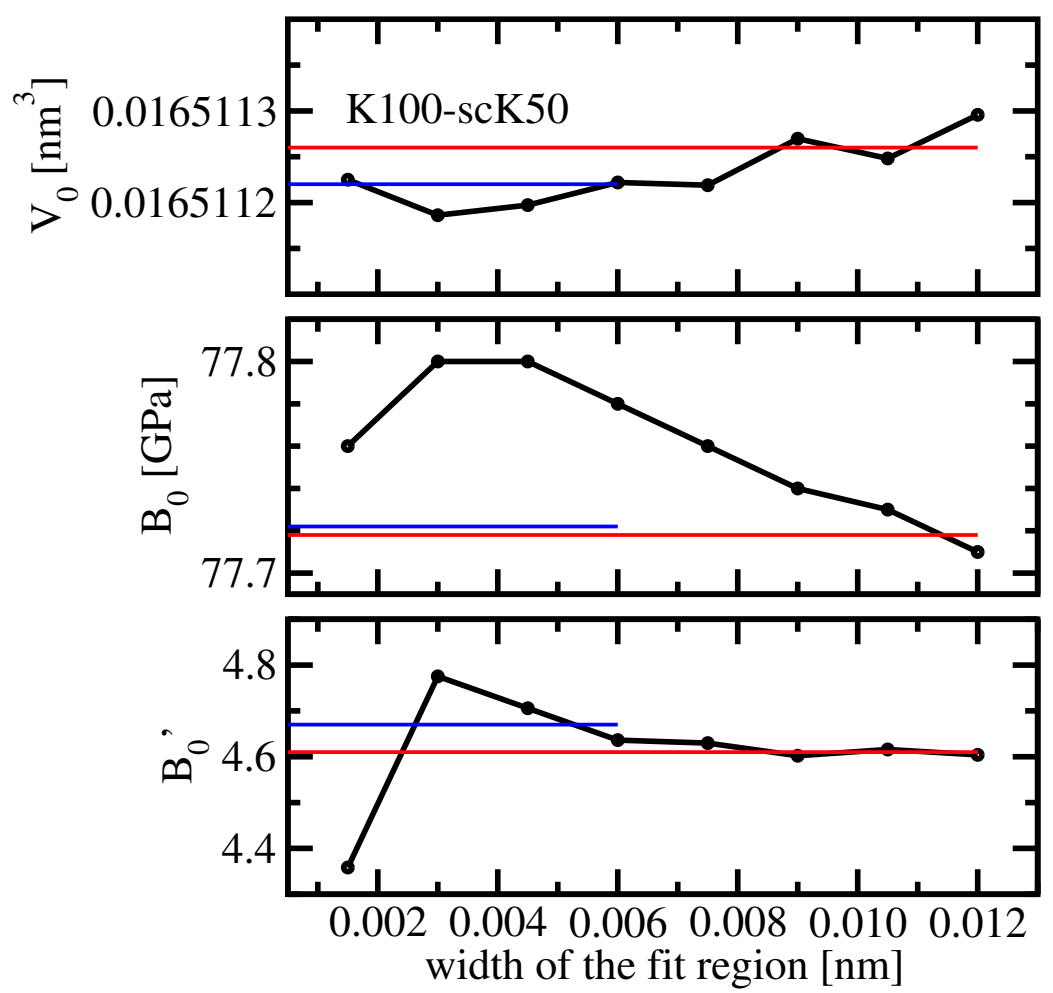

Figure 8. Results of third-order Birch-Murnaghan fits to few data points. The equilibrium volume $V_{0}$ (upper panel), the equilibrium bulk modulus $B_{0}$ (middle panel), and its pressure-derivative $B_{0}^{\prime}$ (lower panel) were obtained from FPLO total energy data for K100-scK50 setting. The circles connected with black lines show results obtained from four $E(a)$ data points, centered at the equilibrium lattice parameter and equally distributed over the fit region. The red (blue) lines show fit results obtained from 25 (13) $E(a)$ data points equally distributed within $0.012(0.006) \mathrm{nm}$ around the equilibrium lattice parameter.

The possibility to use a minimum number of precise data points to numerically evaluate quantities derived from the total energy can be useful e.g. for screening studies. However, this chance comes at the price of a sufficiently dense $k$-mesh. As long as specific 
effects of band structure or DOS, such as ETTs, Weyl points or magnetic instabilities, are not important, it can be useful to apply broadening techniques with a less dense $k$-mesh. They offer the advantages to reduce the related effort and to smoothen the selfconsistent iteration.

\section{Summary}

Electronic structure calculation packages such as the famous Wien2k code, developed and maintained by Karlheinz Schwarz and his group for decades, are meanwhile indispensable tools for the interpretation of experimental data as well as for the prediction of materials properties. In the latter case, there is no standard to gauge the result but by other codes.

Doing so, we have confirmed the classification of Wien2k as the "gold standard" concerning total energy accuracy. This high numerical accuracy is achieved, as in the case of similar codes [1], by using a large and elaborated basis set for the representation of the Bloch wave function. The FPLO code, taken in this study for comparison, uses a smaller basis set. In consequence, it can only reach slightly lower total energy accuracy than Wien2k, but it has the advantage of considerably smaller numerical effort.

Either of the two compared codes allows for the detection of one of the known electronic topological transitions of Aluminium under pressure by appropriate evaluation of $d^{3} E / d a^{3}(a)$. The other two discussed ETT, which are of different topological nature than the former one, are only visible in the density of states and in the Fermi surface. A surprising finding is the high total energy precision of FPLO, demonstrated by comparing $d^{3} E / d a^{3}$ with related Wien2k data.

The total energy precision can be improved in both codes by refining the $k$-mesh. While this behavior is expected, further strong improvements of the numerical precision are obtained in the case of Wien2k by optimizing the value of $R k_{\max }$ and in the case of FPLO by modifying a real-space integration mesh. Thus, it also became possible to perform meaningful third-order Birch-Murnaghan fits to the minimum number of four data points of the obtained very precise $E(V)$.

Concerning both codes, a counterintuitive finding has to be mentioned: tuning the respective basis sets toward optimum numerical accuracy results in a reduced numerical precision. This feature presumably originates from incipient linear dependence of the basis functions.

Finally, we note that our approach to quantify the numerical precision by means of total energy derivatives is not only applicable to the two codes considered in the present study, but can easily be extended to other methods.

Author Contributions: Conceptualization and methodology, M.R., A.M. and H.R.; software, K.K.; investigation, all authors; writing — original draft preparation, M.R. and K.K.; writing —review and editing, all authors. All authors have read and agreed to the published version of the manuscript.

Funding: This research received no external funding.

Data Availability Statement: The data presented in this study are available on request from the corresponding author.

Acknowledgments: We are are grateful to Ulrike Nitzsche for her efforts in establishing and maintaining the IFW high-performance computational environment.

Conflicts of Interest: The authors declare no conflict of interest.

\section{References}

1. Lejaeghere, K.; Bihlmayer, G.; Björkman, T.; Blaha, P.; Blügel, S.; Blum, V.; Caliste, D.; Castelli, I.E.; Clark, S.J.; Dal Corso, A.; et al. Reproducibility in density functional theory calculations of solids. Science 2016, 351, aad3000. [CrossRef] [PubMed]

2. Accuracy and Precision. Available online: https:/ / en.wikipedia.org/wiki/Accuracy_and_precision (accessed on 5 January 2022).

3. Eschrig, H. Optimized LCAO Method and the Electronic Structure of Extended Systems; Akademie-Verlag: Berlin, Germany, 1988.

4. Schwarz, K.; Blaha, P. Solid state calculations using WIEN2k. Comput. Mater. Sci. 2003, 28, 259-273. [CrossRef] 
5. Koepernik, K.; Eschrig, H. Full-potential nonorthogonal local-orbital minimum-basis band-structure scheme. Phys. Rev. B 1999, 59, 1743. [CrossRef]

6. Occelli, F.; Farber, D.L.; Badro, J.; Aracne, C.M.; Teter, D.M.; Hanfland, M.; Canny, B.; Couzinet, B. Experimental Evidence for a High-Pressure Isostructural Phase Transition in Osmium. Phys. Rev. Lett. 2004, 93, 095502. [CrossRef] [PubMed]

7. Koudela, D.; Richter, M.; Möbius, A.; Koepernik, K.; Eschrig, H. Lifshitz transitions and elastic properties of Osmium under pressure. Phys. Rev. B 2006, 74, 214103. [CrossRef]

8. Harrison, W.A. Fermi Surface in Aluminum. Phys. Rev. 1959, 116, 555. [CrossRef]

9. Joss, W.; Monnier, R. Fermi surface of aluminium under homogeneous strain. J. Phys. F Met. Phys. 1980, 10, 9. [CrossRef]

10. Meissner, T.; Goh, S.K.; Haase, J.; Richter, M.; Koepernik, K.; Eschrig, H. Nuclear magnetic resonance at up to 10.1 GPa pressure detects an electronic topological transition in aluminum metal. J. Phys. Condens. Matter 2014, 26, 015501. [CrossRef] [PubMed]

11. Available online: https:/ / www.fplo.de/ (accessed on 5 January 2022).

12. Koelling, D.D.; Harmon, B.N. A technique for relativistic spin-polarised calculations. J. Phys. C Solid State Phys. 1977, 10, 3107. [CrossRef]

13. Perdew, J.P.; Burke, K.; Ernzerhof, M. Generalized Gradient Approximation Made Simple. Phys. Rev. Lett. 1996, 77, 3865. [CrossRef] [PubMed]

14. Blöchl, P.; Jepsen, O.; Andersen, O.K. Improved tetrahedron method for Brillouin-zone integrations. Phys. Rev. B 1994, $49,16223$. [CrossRef] [PubMed]

15. Blanter, Y.M.; Kaganov, M.I.; Pantsulaya, A.V.; Varlamov, A.A. The theory of electronic topological transitions. Phys. Rep. 1994, 245, 159. [CrossRef]

16. Rosner, H.; Koudela, D.; Schwarz, U.; Handstein, A.; Hanfland, M.; Opahle, I.; Koepernik, K.; Kuz'min, M.; Müller, K.H.; Mydosh, J.; et al. Magneto-elastic lattice collapse in YCo5. Nat. Phys. 2006, 2, 469-472. [CrossRef]

17. O’Bannon, E.; Söderlind, P.; Sneed, D.; Lipp, M.; Cynn, H.; Smith, J.; Park, C.; Jenei, Z. High pressure stability of $\beta$-Zr: No evidence for isostructural phase transitions. High Press. Res. 2021, 41, 247-266. [CrossRef] 